\title{
Frontal polymerization in the presence of an inert material
}

\author{
D.M.G. COMISSIONG ${ }^{1}$, L.K. GROSS ${ }^{2}$ and V.A. VOLPERT ${ }^{1}$ \\ ${ }^{1}$ Department of Engineering Sciences and Applied Mathematics, Northwestern University, Evanston IL 60208-3125, \\ USA; ${ }^{2}$ Department of Theoretical and Applied Mathematics, The University of Akron, Akron, OH 44325-4002, \\ USA. (E-mail:v-volpert@northwestern.edu)
}

Received 15 April 2005; accepted in revised form 2 December 2005 / Published online: 21 February 2006

\begin{abstract}
Propagation of a one-dimensional polymerization wave in a sandwich-type two-layer setting, where one layer is reactive while the other layer consists of an inert material, is considered. Heat exchange is possible between the two layers and thus the presence of the inert layer can significantly affect propagation of the polymerization wave. The existence of multiple propagating fronts in the system for the same parameter values is demonstrated. Linear stability analysis of the propagating fronts is performed, and conditions for oscillatory propagation of the fronts are determined.
\end{abstract}

Key words: frontal polymerization, linear stability, sandwich model

\section{Introduction}

Frontal polymerization (FP) is a chemical process whereby monomer is converted to polymer via a localized reaction zone. The front propagates as a result of the coupling of thermal diffusion and exothermic Arrhenius reaction kinetics. Frontal polymerization was first discovered experimentally in 1972 [1]. It is a simple technique which can be easily implemented on a laboratory scale, and has been the subject of intense experimental and theoretical investigation in recent years (see, e.g. [2] and the references therein). The localized reaction zones and rapid increase in temperature across the reaction wave are features that distinguish FP from conventional polymerization methods. The interest in FP stems from its untapped potential for the production of novel materials. Uniform composites, hydrogels, simultaneous interpenetrating networks, copolymers, polymer blends and functional gradient materials can be listed among the many applications being considered for industrial production via FP.

We consider the steady propagation of a one-dimensional frontal polymerization (FP) wave in a sandwich-type two-layer model. One layer is reactive. It contains a mixture of a monomer and initiator. The other layer consists of an inert material. The two layers can exchange heat and thus the presence of the inert layer can significantly affect propagation of the polymerization wave through the reactive layer. Our mathematical model is a generalization of a simpler model proposed in [3] in the context of combustion studies. A single stationary solution is found for the reactive layer in the presence of very thin inert layers. As the thickness of the inert layer is increased (this corresponds to a change in the inter-layer heat-exchange parameters), a saddle node bifurcation occurs and two new steady states are formed. Another turning point is found as the thickness of the inert layer exceeds another critical point, thereby reducing the system once more to a single stationary state. The existence of more than one stable basic solution creates the opportunity for unpredictable jumps from one solution to the next. This form of hysteresis may be undesirable in manufacturing processes, and must be properly understood before it can be controlled. We proceed to carry 
out a linear stability analysis of the uniformly propagating waves. For systems that exhibit multiple steady states, linear stability analysis is a crucial first step for the accurate prediction of the behavior of the system when subject to small perturbations from its basic state.

\section{Mathematical model}

We consider two adjacent thin layers. One layer is made up of an inert material, and the other layer is a reactive mixture, which initially consists of monomer and initiator. There is thermal contact between the two layers. The polymerization process occurring in the reactive layer is exothermic, and therefore there is heat exchange between the layers as illustrated by Figure 1. In order to formulate a mathematical model, we first discuss the polymerization process occurring in the reactive layer.

The polymerization process is the free-radical polymerization which involves a standard sequence of chemical reactions [4, Chapter 3]. The process begins when the initiator decomposes, forming two radicals. Each radical can then combine with a monomer, initiating a polymer chain. A polymer chain grows by combining with another monomer to form a longer chain, and terminates by combining with a radical, either another growing chain or an initiator radical. Thus, the kinetic scheme involves the decomposition step, initiation step, propagation step and the termination step.

These kinetics equations can be simplified by using a steady-state assumption. Indeed, the rate of production and consumption of radicals is much larger than the overall rate of change of the radical concentration, which allows us to reduce the differential equations to algebraic balances. This steady-state assumption has been justified in the context of a frontal polymerization problem in [5], and it reduces the kinetics equations to

$$
\frac{\partial I}{\partial t}+k_{\mathrm{d}} I=0, \quad \frac{\partial M}{\partial t}+k_{e} \sqrt{I} M=0 .
$$

Here $I$ and $M$ are the concentrations of the initiator and the monomer, respectively, $t$ is the time, and $k_{\mathrm{d}}$ and $k_{\mathrm{e}}$ are the decomposition and the polymerization reaction rate parameters which depend on the temperature $T_{\mathrm{r}}$ of the reactive layer. This dependence is given by the Arrhenius law

$$
k_{\mathrm{d}}=k_{\mathrm{d}}^{0} \exp \left\{-E_{\mathrm{d}} /\left(R T_{\mathrm{r}}\right)\right\}, \quad k_{\mathrm{e}}=k_{\mathrm{e}}^{0} \exp \left\{-E_{\mathrm{e}} /\left(R T_{\mathrm{r}}\right)\right\}
$$

where $R$ is the gas constant, $k_{\mathrm{d}}^{0}, k_{\mathrm{e}}^{0}$ and $E_{\mathrm{d}}, E_{\mathrm{e}}$ are the frequency factors and activation energies of the two reactions.

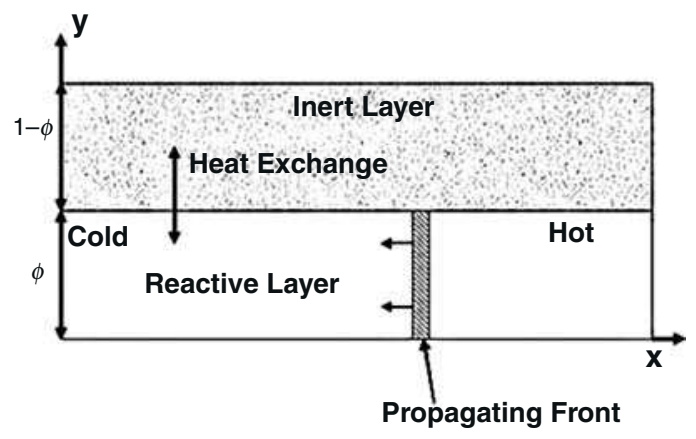

Figure 1. Diagram showing system under study. 
Next, we need to formulate the energy balance in the reactive layer. Since the main heat producing step is the propagation step [6], the heat equation has the form

$$
\phi c_{\mathrm{r}} \rho_{\mathrm{r}} \frac{\partial T_{\mathrm{r}}}{\partial t}=\phi \lambda_{\mathrm{r}} \frac{\partial^{2} T_{\mathrm{r}}}{\partial x^{2}}-\phi \Delta H k_{\mathrm{e}} \sqrt{I} M-\alpha\left(T_{\mathrm{r}}-T_{\mathrm{i}}\right) .
$$

Here $\lambda_{\mathrm{r}}$ is the thermal conductivity, $\Delta H$ is the enthalpy of the reaction, $c_{\mathrm{r}}$ is the specific heat and $\rho_{\mathrm{r}}$ is the mixture density. Next, $T_{\mathrm{i}}$ is the temperature of the inert layer, and $\phi$ is the ratio of the thickness of the reactive layer to the total thickness of the two layers. The last term of the heat-balance equation describes heat exchange between the two layers. The heat balance in the inert layer is given by the equation

$$
(1-\phi) c_{\mathrm{i}} \rho_{\mathrm{i}} \frac{\partial T_{\mathrm{i}}}{\partial t}=(1-\phi) \lambda_{\mathrm{i}} \frac{\partial^{2} T_{\mathrm{i}}}{\partial x^{2}}-\alpha\left(T_{\mathrm{i}}-T_{\mathrm{r}}\right) .
$$

In a fixed coordinate frame $(\widetilde{x}),-\infty<\widetilde{x}<\infty$, the front propagates along the $\widetilde{x}$-axis in the direction of decreasing $\tilde{x}$. By introducing a moving coordinate system $x=\tilde{x}-\varphi(t)$ where $\varphi$ is the location of the reaction front at time $t$, we fix the front at $x=0$. Thus, $\mathrm{d} \varphi(t) / \mathrm{d} t<0$ is the velocity of the propagating front.

We rewrite the equations in the moving coordinate system and further simplify the problem making use of the fact that the activation energies of the decomposition and polymerization reactions are large, which results in narrow reaction zones. In the limit of infinite activation energy, the reaction zone shrinks to a moving surface, termed a front. In this case the equations must be solved without the reaction term both ahead of and behind the reaction front and matched at the reaction front by satisfying matching conditions. Thus, we solve the reactionless equations

$$
\begin{aligned}
& \frac{\partial I}{\partial t}-\frac{\mathrm{d} \varphi}{\mathrm{d} t} \frac{\partial I}{\partial x}=0, \quad \frac{\partial M}{\partial t}-\frac{\mathrm{d} \varphi}{\mathrm{d} t} \frac{\partial M}{\partial x}=0, \\
& \frac{\partial T_{\mathrm{r}}}{\partial t}-\frac{\mathrm{d} \varphi}{\mathrm{d} t} \frac{\partial T_{\mathrm{r}}}{\partial x}=\kappa_{\mathrm{r}} \frac{\partial^{2} T_{\mathrm{r}}}{\partial x^{2}}-\alpha_{\mathrm{r}}\left(T_{\mathrm{r}}-T_{\mathrm{i}}\right) \\
& \frac{\partial T_{\mathrm{i}}}{\partial t}-\frac{\mathrm{d} \varphi}{\mathrm{d} t} \frac{\partial T_{\mathrm{i}}}{\partial x}=\kappa_{\mathrm{i}} \frac{\partial^{2} T_{\mathrm{i}}}{\partial x^{2}}-\alpha_{\mathrm{i}}\left(T_{\mathrm{i}}-T_{\mathrm{r}}\right)
\end{aligned}
$$

both ahead of $(x<0)$ and behind $(x>0)$ the front. Here

$$
\kappa_{\mathrm{r}}=\frac{\lambda_{\mathrm{r}}}{c_{\mathrm{r}} \rho_{\mathrm{r}}}, \quad \kappa_{\mathrm{i}}=\frac{\lambda_{\mathrm{i}}}{c_{\mathrm{i}} \rho_{\mathrm{i}}}, \quad \alpha_{\mathrm{r}}=\frac{\alpha}{c_{\mathrm{r}} \rho_{\mathrm{r}} \phi}, \quad \alpha_{\mathrm{i}}=\frac{\alpha}{c_{\mathrm{i}} \rho_{\mathrm{i}}(1-\phi)} .
$$

Boundary conditions far ahead of the front describe the initial state of the layers:

$$
x=-\infty: \quad T_{\mathrm{r}}=T_{\mathrm{i}}=T_{0}, \quad M=M_{0}, \quad I=I_{0}
$$

whereas far behind the front the final state is

$$
x=+\infty: \quad T_{\mathrm{r}}=T_{\mathrm{i}}=T_{\mathrm{f}} .
$$

This last condition states that far behind the reaction front the reactive temperature goes to a constant, and that because of the heat exchange between the layers the inert temperature takes on the same value $T_{\mathrm{f}}$; this value $T_{\mathrm{f}}$ of the final temperature is however unknown, and has to be determined in the course of solution of the problem. 
The matching conditions have the form

$$
\begin{aligned}
& {\left[T_{\mathrm{r}}\right]=0, \quad\left[T_{\mathrm{i}}\right]=0, \quad \kappa_{\mathrm{r}}\left[\frac{\partial T_{\mathrm{r}}}{\partial x}\right]=q\left(M_{0}-M_{\mathrm{b}}\right) \frac{\mathrm{d} \varphi}{\mathrm{d} t}, \quad \kappa_{\mathrm{i}}\left[\frac{\partial T_{\mathrm{i}}}{\partial x}\right]=0,} \\
& \left(\frac{\mathrm{d} \varphi}{\mathrm{d} t}\right)^{2}=F\left(T_{\mathrm{b}}\right), \quad M_{\mathrm{b}}=M_{0} \exp \left(-j_{0}\right) .
\end{aligned}
$$

The brackets denote a jump in a quantity across the front

$$
[v]=\left.v\right|_{x=0^{+}}-\left.v\right|_{x=0^{-}} .
$$

Next, $T_{\mathrm{b}}$ and $M_{\mathrm{b}}$ are the temperature and monomer concentration at the front (i.e., at $x=0$ ), respectively, $q=-\Delta H /\left(c_{\mathrm{r}} \rho_{\mathrm{r}}\right)$ is the temperature increase per unit concentration of the reacted monomer, and the function $F$ is given by

$$
\begin{aligned}
& F\left(T_{\mathrm{b}}\right)=\frac{\kappa_{\mathrm{r}} k_{\mathrm{d}}^{0} R T_{\mathrm{b}}^{2}}{2 q M_{0} E_{\mathrm{d}}} \exp \left(j_{0}-\frac{E_{\mathrm{d}}}{R T_{b}}\right)\left(\int_{0}^{j_{0}} \frac{\mathrm{e}^{\eta}-1}{\eta} \mathrm{d} \eta\right)^{-1}, \\
& j_{0} \equiv 2 \sqrt{I_{0}} \frac{k_{\mathrm{e}}^{0}}{k_{\mathrm{d}}^{0}} \exp \frac{E_{\mathrm{d}}-E_{\mathrm{e}}}{R T_{\mathrm{b}}} .
\end{aligned}
$$

We remark that the above treatment of the polymerization problem assumes that both decomposition and polymerization reactions occur in the same reaction zone. This is indeed the case for typical values of the kinetic parameters of the polymerization processes. In general, it does not have to be the case, i.e., different reactions can occur at different spatial locations as known from combustion literature [7-9]. Details of the derivation of the matching conditions (9), (10) can be found, e.g., in [10].

\section{Steady-state analysis}

In this section we determine stationary solutions of the above problem, which correspond to uniformly propagating one-dimensional traveling waves in the original problem. Our preliminary objective is to determine the effect that the inert layer has on the basic state of the propagating reaction front.

We solve the following reactionless system ahead of $(x<0)$ and behind $(x>0)$ the front

$$
\begin{aligned}
& \frac{\mathrm{d} \widehat{I}}{\mathrm{~d} x}=0, \quad \frac{\mathrm{d} \widehat{M}}{\mathrm{~d} x}=0, \\
& \kappa_{\mathrm{r}} \frac{\mathrm{d}^{2} \widehat{T}_{\mathrm{r}}}{\mathrm{d} x^{2}}-\widehat{u} \frac{\mathrm{d} \widehat{T}_{\mathrm{r}}}{\mathrm{d} x}-\alpha_{\mathrm{r}}\left(\widehat{T}_{\mathrm{r}}-\widehat{T}_{\mathrm{i}}\right)=0, \quad \kappa_{\mathrm{i}} \frac{\mathrm{d}^{2} \widehat{T}_{\mathrm{i}}}{\mathrm{d} x^{2}}-\widehat{u} \frac{\mathrm{d} \widehat{T}_{\mathrm{i}}}{\mathrm{d} x}-\alpha_{\mathrm{i}}\left(\widehat{T}_{\mathrm{i}}-\widehat{T}_{\mathrm{r}}\right)=0
\end{aligned}
$$

subject to the boundary conditions

$$
\begin{array}{ll}
x=-\infty: & \widehat{T}_{\mathrm{r}}=\widehat{T}_{\mathrm{i}}=T_{0}, \quad \widehat{M}=M_{0}, \quad \widehat{I}=I_{0}, \\
x=+\infty: & \widehat{T}_{\mathrm{r}}=\widehat{T}_{\mathrm{i}}=\widehat{T}_{\mathrm{f}},
\end{array}
$$

and the matching conditions

$$
\left[\widehat{T}_{\mathrm{r}}\right]=0, \quad\left[\widehat{T}_{\mathrm{i}}\right]=0, \quad \kappa_{\mathrm{r}}\left[\frac{\mathrm{d} \widehat{T}_{\mathrm{r}}}{\mathrm{d} x}\right]=-q \widehat{u}\left(M_{0}-\widehat{M}_{\mathrm{b}}\right), \quad \kappa_{\mathrm{i}}\left[\frac{\mathrm{d} \widehat{T}_{\mathrm{i}}}{\mathrm{d} x}\right]=0,
$$




$$
\widehat{u}^{2}=F\left(\widehat{T}_{\mathrm{b}}\right), \quad \widehat{M}_{\mathrm{b}}=M_{0} \exp \left(-\widehat{j}_{0}\right), \quad \widehat{j_{0}} \equiv 2 \sqrt{I_{0}} \frac{k_{\mathrm{e}}^{0}}{k_{\mathrm{d}}^{0}} \exp \frac{E_{\mathrm{d}}-E_{\mathrm{e}}}{R \widehat{T}_{\mathrm{b}}} .
$$

Here the quantities with the hats denote the stationary solution, and $\widehat{u}$ is the speed of the uniformly propagating wave. The stationary solution that satisfies (11)-(17) is given by

$$
\begin{aligned}
& \widehat{M}(x)=\left\{\begin{array}{ll}
M_{0}, & x<0 \\
\widehat{M}_{b}, & x>0
\end{array}, \quad \widehat{I}(x)=\left\{\begin{array}{ll}
I_{0}, & x<0 \\
0, & x>0
\end{array},\right.\right. \\
& \widehat{T}_{\mathrm{r}}(x)=\left\{\begin{array}{ll}
T_{0}+q\left(M_{0}-\widehat{M}_{\mathrm{b}}\right)\left\{C_{2} \exp \left(\lambda_{2} x\right)+C_{3} \exp \left(\lambda_{3} x\right)\right\}, & x<0 \\
\widehat{T}_{\mathrm{f}}+q\left(M_{0}-\widehat{M}_{\mathrm{b}}\right) C_{1} \exp \left(\lambda_{1} x\right), & x>0
\end{array},\right. \\
& \widehat{T}_{\mathrm{i}}(x)=\left\{\begin{array}{ll}
T_{0}+q\left(M_{0}-\widehat{M}_{\mathrm{b}}\right)\left\{B_{2} \exp \left(\lambda_{2} x\right)+B_{3} \exp \left(\lambda_{3} x\right)\right\}, & x<0 \\
\widehat{T}_{\mathrm{f}}+q\left(M_{0}-\widehat{M}_{\mathrm{b}}\right) B_{1} \exp \left(\lambda_{1} x\right), & x>0
\end{array} .\right.
\end{aligned}
$$

Here $\lambda_{1}, \lambda_{2}$, and $\lambda_{3}$ are the roots of the characteristic equation

$$
\kappa_{\mathrm{r}} \kappa_{\mathrm{i}} \lambda^{3}-\widehat{u}\left(\kappa_{\mathrm{r}}+\kappa_{\mathrm{i}}\right) \lambda^{2}+\left(\widehat{u}^{2}-\alpha_{\mathrm{r}} \kappa_{\mathrm{i}}-\alpha_{\mathrm{i}} \kappa_{\mathrm{r}}\right) \lambda+\widehat{u}\left(\alpha_{\mathrm{r}}+\alpha_{\mathrm{i}}\right)=0 .
$$

It can be shown that this equation has three real roots for all $\alpha_{\mathrm{r}}>0, \alpha_{\mathrm{i}}>0, \kappa_{\mathrm{r}}>0, \kappa_{\mathrm{i}}>0$, $\widehat{u}>0$. Moreover, one of these roots is always negative (we denote it by $\lambda_{1}$ ), while the other two roots are always positive (we denote them by $\lambda_{2}$ and $\lambda_{3}$ ). It follows from the fact that the cubic parabola on the left-hand side of (22) has a positive coefficient of the cubic term, is positive at $\lambda=0$ and negative at $\lambda=\widehat{u} / \kappa_{\mathrm{r}} \cdot \max \left\{1, \kappa_{\mathrm{r}} / \kappa_{\mathrm{i}}\right\}$, which can be verified directly. Next, the final temperature $\widehat{T}_{f}$ and the constants $C_{1}, C_{2}, C_{3}$ and $B_{1}, B_{2}, B_{3}$ are given by

$$
\begin{aligned}
& \widehat{T}_{\mathrm{f}}=T_{0}+q\left(M_{0}-\widehat{M}_{\mathrm{b}}\right) \frac{\alpha_{\mathrm{i}}^{0}}{\alpha_{\mathrm{i}}^{0}+\kappa^{0} \alpha_{\mathrm{r}}^{0}}, \quad C_{1}=-\frac{\alpha_{\mathrm{r}}^{0}+\left(\nu_{3}-1\right)\left(\nu_{2}-1\right)}{\nu_{1}\left(\nu_{1}-\nu_{3}\right)\left(\nu_{1}-\nu_{2}\right)}, \\
& C_{2}=-\frac{\alpha_{\mathrm{r}}^{0}+\left(\nu_{3}-1\right)\left(\nu_{1}-1\right)}{v_{2}\left(v_{1}-v_{2}\right)\left(v_{2}-v_{3}\right)}, \quad C_{3}=\frac{\alpha_{\mathrm{r}}^{0}+\left(\nu_{1}-1\right)\left(\nu_{2}-1\right)}{\nu_{3}\left(\nu_{2}-v_{3}\right)\left(\nu_{1}-v_{3}\right)}, \\
& B_{j}=\left(1-\frac{v_{j}^{2}-v_{j}}{\alpha_{\mathrm{r}}^{0}}\right) C_{j}, \quad j=1,2,3 .
\end{aligned}
$$

Here

$$
\kappa^{0}=\frac{\kappa_{\mathrm{i}}}{\kappa_{\mathrm{r}}}, \quad \alpha_{\mathrm{r}}^{0}=\frac{\kappa_{\mathrm{r}}}{\widehat{u}^{2}} \alpha_{\mathrm{r}}, \quad \alpha_{\mathrm{i}}^{0}=\frac{\kappa_{\mathrm{i}}}{\widehat{u}^{2}} \alpha_{\mathrm{i}},
$$

and $v_{j}(j=1,2,3)$ are nondimensional eigenvalues $\lambda_{j}(j=1,2,3)$,

$$
v_{j}=\frac{\kappa_{\mathrm{r}}}{\widehat{u}} \lambda_{j}
$$

which satisfy the equation

$$
\kappa^{0} v^{3}-\left(1+\kappa^{0}\right) v^{2}+\left(1-\alpha_{\mathrm{i}}^{0} / \kappa^{0}-\alpha_{\mathrm{r}}^{0} \kappa^{0}\right) v+\alpha_{\mathrm{r}}^{0}+\alpha_{\mathrm{i}}^{0} / \kappa^{0}=0 .
$$

The first conclusion concerning the structure of the solution can be drawn from these preliminary equations. We observe that the constants $C_{1}$ and $B_{1}$ can be written in the form

$$
C_{1}=-\frac{\alpha_{\mathrm{r}}^{0}}{\kappa^{0}} \frac{\kappa^{0} \nu_{1}-1}{\nu_{1}\left(\nu_{1}-1\right)\left(\nu_{1}-\nu_{2}\right)\left(\nu_{1}-\nu_{3}\right)}>0, \quad B_{1}=-\frac{\alpha_{\mathrm{i}}^{0}}{\kappa^{0} \alpha_{\mathrm{r}}^{0}} \frac{\nu_{1}-1}{\kappa^{0} \nu_{1}-1} C_{1}<0 .
$$




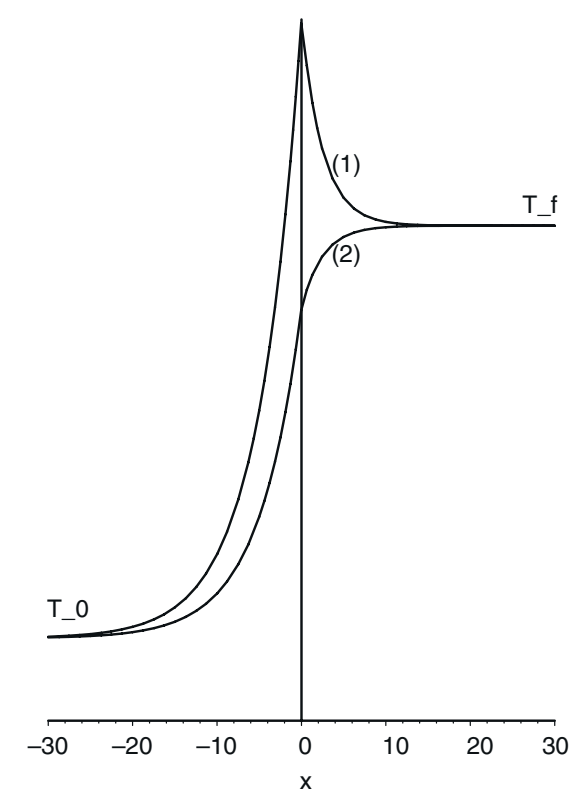

Figure 2. Schematic of the temperature distribution in the problem. Here the front is located at $x=0$. The temperature of the reactive layer $T_{\mathrm{r}}$ (labeled by (1)) has a maximum at the front, while the temperature of the inert layer (labeled by (2)) is a monotone function.

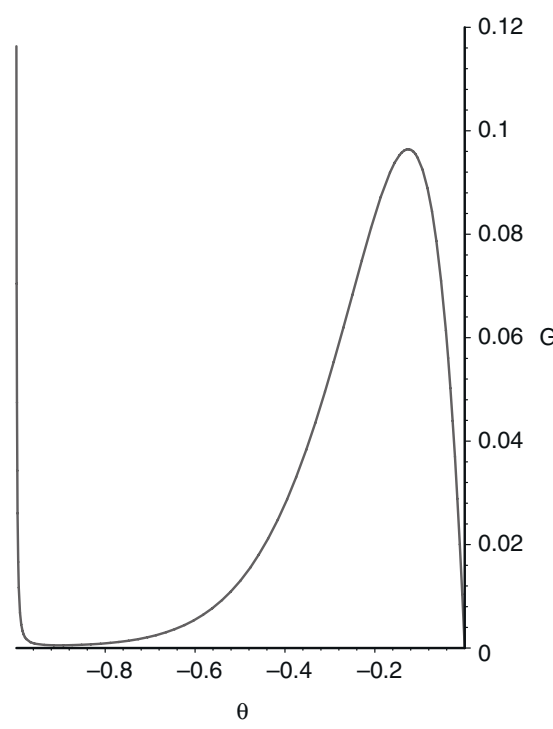

Figure 3. The graph of the function $G(\theta)$ in (30).

The signs immediately follow from the signs of $v_{1}, v_{2}$, and $\nu_{3}$. This means that $T_{\mathrm{r}}$ increases as $x$ goes from $-\infty$ to zero (i.e., ahead of the front), and then decreases for $x>0$, i.e., behind the front, while $T_{i}$ is a monotonically increasing function everywhere as illustrated by Figure 2.

In order to complete the solution of the steady-state problem we have to determine the propagation velocity $\widehat{u}$ and the temperature $\widehat{T}_{\mathrm{b}}$ at the reaction front. The equations that relate these two quantities are

$$
\widehat{u}^{2}=F\left(\widehat{T}_{\mathrm{b}}\right)
$$

and

$$
\widehat{T}_{\mathrm{b}}=\widehat{T}_{\mathrm{f}}+q\left(M_{0}-\widehat{M}_{\mathrm{b}}\right) C_{1},
$$

which follows from the definition that $\widehat{T}_{\mathrm{b}}$ is the temperature at the front $(x=0)$ and solution (20). Nondimensionalizing Equations (24), (25) and using

$$
\widehat{M}_{\mathrm{b}}=\exp \left(-\widehat{j}_{0}\right)
$$

we obtain

$$
\begin{aligned}
& \widehat{u}^{2}=u_{\mathrm{a}}^{2}(1+\sigma \theta)^{2} \exp \left(\frac{Z_{\mathrm{e}} \theta}{1+\sigma \theta}\right)\left(\widehat{j_{0}} \mathrm{e}^{-\widehat{j}_{0}} \int_{0}^{\widehat{j_{0}}} \frac{\exp (\eta)-1}{\eta} \mathrm{d} \eta\right)^{-1}, \\
& \frac{\theta+1}{1-\mathrm{e}^{-\hat{j}_{0}}}=\frac{\alpha_{\mathrm{i}}^{0}}{\alpha_{\mathrm{i}}^{0}+\kappa^{0} \alpha_{\mathrm{r}}^{0}}+\frac{\alpha_{\mathrm{r}}^{0}}{\kappa^{0}} \frac{1-\kappa^{0} \nu_{1}}{v_{1}\left(v_{1}-1\right)\left(v_{1}-v_{2}\right)\left(v_{1}-v_{3}\right)} .
\end{aligned}
$$


Here $\theta$ is the nondimensional temperature at the front,

$$
\theta=\frac{\widehat{T}_{\mathrm{b}}-T_{\mathrm{a}}}{q M_{0}},
$$

where $T_{\mathrm{a}}=T_{0}+q M_{0}$ is the adiabatic temperature in the reactive layer (i.e., in the case that no heat exchange with the inert layer is allowed). Note that the right-hand side of (28) implicitly depends on $\widehat{u}$ through the constants $\alpha_{\mathrm{i}}^{0}$ and $\alpha_{\mathrm{r}}^{0}$ as well as the nondimensional characteristic roots $v$. Next, the function $\widehat{j_{0}}$ in (27), (28), which is given by (18), and which can be understood as a measure of incompleteness of the chemical conversion (see (26)), can be written in the form

$$
\widehat{j_{0}}=j_{*} \exp \left(-\frac{Z_{\mathrm{de}} \theta}{1+\sigma \theta}\right), \quad j_{*}=2 \sqrt{I_{0}} \frac{k_{\mathrm{e}}^{0}}{k_{\mathrm{d}}^{0}} \exp \left(\frac{E_{\mathrm{d}}-E_{\mathrm{e}}}{R T_{\mathrm{a}}}\right) .
$$

Note that $\widehat{j_{0}}>j_{*}$ and the $\widehat{j_{0}}$-dependent factor in (27) goes to one as $\widehat{j_{0}} \rightarrow \infty$, while $\theta \rightarrow 0$ in this limit. As a result, $\widehat{u}$ goes to the adiabatic propagation velocity $u_{\mathrm{a}}$ that occurs in case of complete conversion and is given by

$$
u_{\mathrm{a}}^{2}=\frac{\kappa_{\mathrm{r}} R T_{\mathrm{a}}}{q M_{0} E_{\mathrm{d}}} \sqrt{I_{0}} k_{\mathrm{e}}^{0} \exp \left(-\frac{E_{\mathrm{e}}}{R T_{\mathrm{a}}}\right)
$$

Finally,

$$
\sigma=\frac{q M_{0}}{T_{\mathrm{a}}}, \quad Z_{\mathrm{e}}=\frac{E_{\mathrm{e}} q M_{0}}{R T_{\mathrm{a}}^{2}}, \quad Z_{\mathrm{de}}=\frac{\left(E_{\mathrm{d}}-E_{\mathrm{e}}\right) q M_{0}}{R T_{\mathrm{a}}^{2}},
$$

with $Z_{\mathrm{e}}, Z_{\mathrm{de}}$ being analogous to the Zeldovich number that is used in combustion theory.

We remark that Equation (27) is a meaningful way to represent the propagation velocity as a function of the front temperature. It clearly shows the effect of different factors on the velocity. Indeed, first it involves the adiabatic velocity $u_{\mathrm{a}}$ that would occur if the chemical conversion were complete $\left(\widehat{M}_{\mathrm{b}}=0\right)$ and there was no heat exchange with the other layer. Next, there is a $\theta$-dependent factor that is responsible for the difference between the actual velocity and the adiabatic velocity due to the presence of heat exchange (i.e., $\theta<0$ in the problem). Finally, the $\widehat{j_{0}}$-dependent factor demonstrates the deviation of the velocity from the adiabatic due to incomplete conversion.

To determine the nondimensional front temperature $\theta$ we need to solve Equation (28) where the characteristic roots $v$ are solutions of (23) that depend on the parameters of the problem as well as $\widehat{u}$, which is given in terms of $\theta$ by (27). Before we do it, let us consider an instructive limiting case. Suppose $\alpha_{\mathrm{i}}=0$ and $\alpha_{\mathrm{r}}>0$. That means that the reactive layer can lose heat to the inert layer, but there is no heat supply to the reactive layer from the inert layer. In other words, this limiting case is similar to a heat-loss extinction problem that has been studied both in combustion and frontal polymerization [11, 12] settings. In this case the characteristic equation has very simple roots,

$$
v_{1}=\frac{1}{2}\left(1-\sqrt{1+4 \alpha_{\mathrm{r}}^{0}}\right), \quad v_{2}=\frac{1}{\kappa^{0}}, \quad v_{3}=\frac{1}{2}\left(1+\sqrt{1+4 \alpha_{\mathrm{r}}^{0}}\right)
$$

and (28) simplifies to

$$
\frac{\theta+1}{1-\mathrm{e}^{-\widehat{j}_{0}}}=\frac{1}{\sqrt{1+4 \alpha_{\mathrm{r}}^{0}}} .
$$




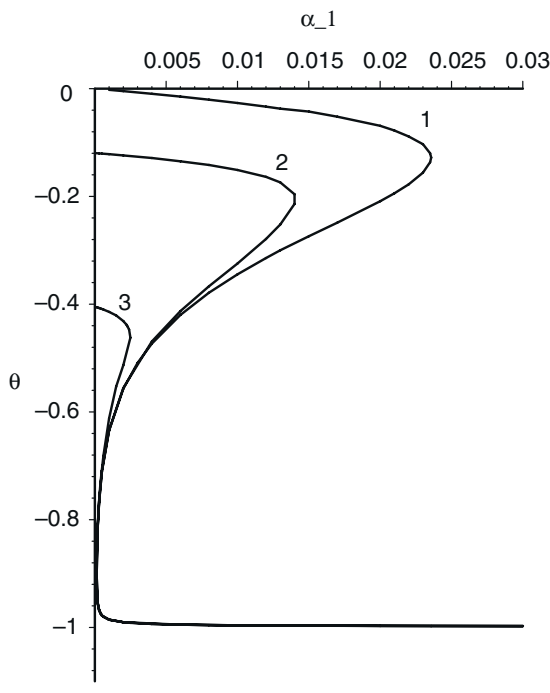

Figure 4. Dependence of the nondimensional front temperature $\theta$ on the heat-transfer coefficient $\alpha_{1}=\alpha_{\mathrm{r}} \kappa_{\mathrm{r}} / u_{\mathrm{a}}^{2}$ for $Z_{\mathrm{e}}=8, Z_{\mathrm{de}}=6, \kappa^{0}=1, u_{\mathrm{a}}=1$, $\alpha_{\mathrm{i}}=0$ and different values of $j_{*}$. Curve $1-j_{*}=20$, curve $2-j_{*}=1$, curve $3-j_{*}=0.05$.

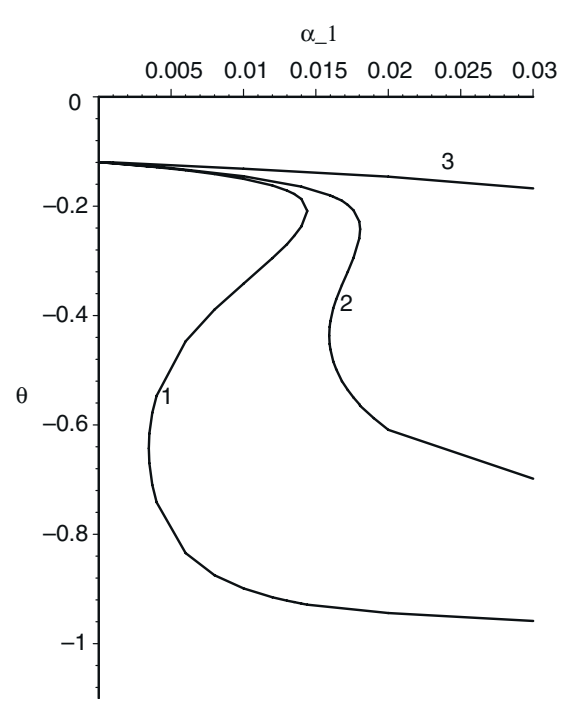

Figure 5. Dependence of the nondimensional front temperature $\theta$ on the heat-transfer coefficient $\alpha_{1}=\alpha_{\mathrm{r}} \kappa_{\mathrm{r}} / u_{\mathrm{a}}^{2}$ for $Z_{\mathrm{e}}=8, Z_{\mathrm{de}}=6, \kappa^{0}=1, u_{\mathrm{a}}=1$, $j_{*}=1$ and different values of the heat-transfer coefficient $\alpha_{2}=\alpha_{\mathrm{i}} \kappa_{\mathrm{i}} / u_{\mathrm{a}}^{2}$. Curve $1-\alpha_{2}=0.001$, curve $2-$ $\alpha_{2}=0 \cdot 01$, curve $3-\alpha_{2}=0 \cdot 1$.

In the limit $j_{*} \gg 1$ we have $\widehat{j_{0}} \gg 1$ so that (27) simplifies to

$$
\frac{\widehat{u}^{2}}{u_{\mathrm{a}}^{2}}=(1+\sigma \theta)^{2} \exp \left(\frac{Z_{\mathrm{e}} \theta}{1+\sigma \theta}\right),
$$

while (29) simplifies to

$$
\frac{\widehat{u}^{2}}{u_{\mathrm{a}}^{2}}=4 \frac{\kappa_{\mathrm{r}} \alpha_{\mathrm{r}}}{u_{\mathrm{a}}^{2}} \frac{(1+\theta)^{2}}{1-(1+\theta)^{2}} .
$$

From the above two equations we obtain an equation for $\theta$ in the form

$$
4 \frac{\kappa_{\mathrm{r}} \alpha_{\mathrm{r}}}{u_{\mathrm{a}}^{2}}=\frac{1-(1+\theta)^{2}}{(1+\theta)^{2}}(1+\sigma \theta)^{2} \exp \left(\frac{Z_{\mathrm{e}} \theta}{1+\sigma \theta}\right) \equiv G(\theta) .
$$

The right-hand side of this equation as a function of $\theta$ is shown in Figure 3 for $Z_{\mathrm{e}}=8$ and $\sigma=0.4$. From this graph we see the critical phenomena in the dependence of $\theta$ on the heatexchange coefficient $\alpha_{\mathrm{r}}$. For $\alpha_{\mathrm{r}}$ greater than a critical value there is only one, low-temperature solution, while for $\alpha_{\mathrm{r}}$ below the critical value there are three solutions for the temperature. This critical behavior is typical of the problem at hand not only in this limiting case but also in general.

We next turn to the case $\alpha_{\mathrm{i}}=0, \alpha_{\mathrm{r}}>0$, but $\hat{j}_{*}$ is not necessarily large. The results are illustrated by Figure 4 . We observe the $S$-shaped dependence of the front temperature on the heat exchange coefficient $\alpha_{\mathrm{r}}$ that degenerates as $\widehat{j}_{*}$ decreases. For even smaller $\widehat{j}_{*}$, i.e., when conversion is substantially incomplete, the solution reduces to a single-valued low-temperature branch. 


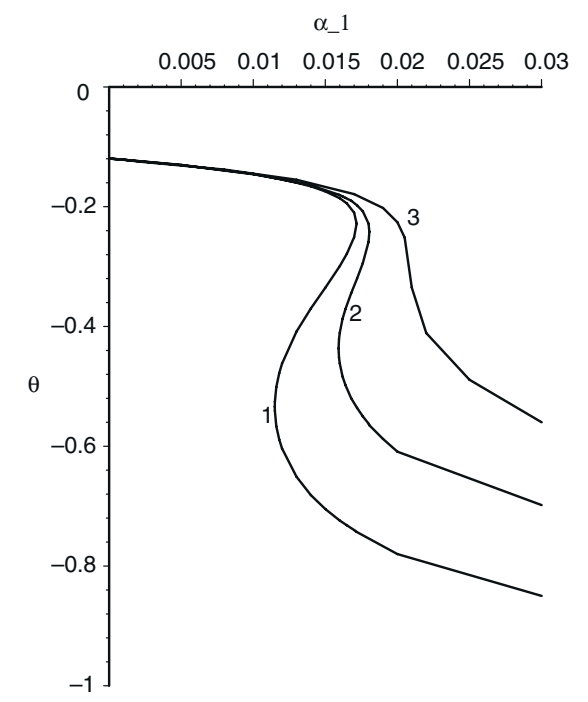

Figure 6. Dependence of the nondimensional front temperature $\theta$ on the heat-transfer coefficient $\alpha_{1}=\alpha_{\mathrm{r}} \kappa_{\mathrm{r}} / u_{\mathrm{a}}^{2}$ for $Z_{\mathrm{e}}=8, Z_{\mathrm{de}}=6, u_{\mathrm{a}}=1, j_{*}=1$, $\alpha_{2}=0.01$ and different values of the thermal-diffusivity ratio $\kappa^{0}$. Here $\kappa^{0}=2$ (curve 1 ), $\kappa^{0}=1$ (curve 2), $\kappa^{0}=0.5$ (curve 3 ).

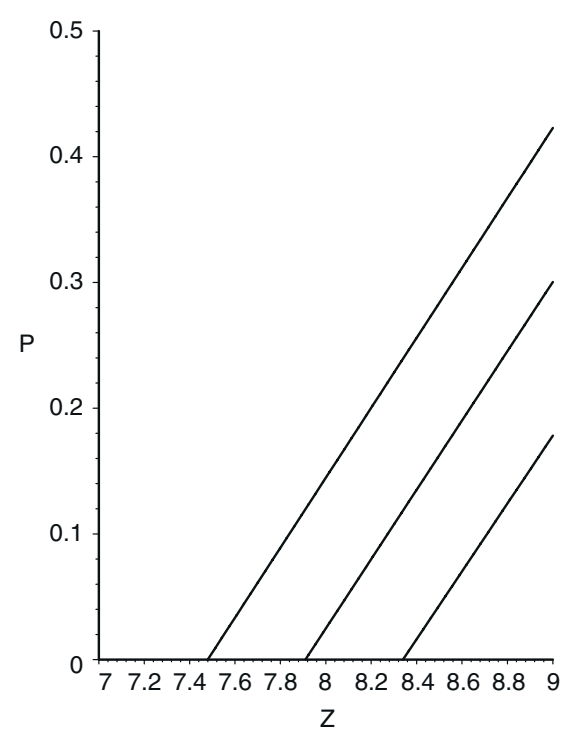

Figure 7. Stability boundaries in the $(Z, P)$-plane for $\kappa^{0}=1, \alpha_{2}=\alpha_{\mathrm{i}} \kappa_{\mathrm{i}} / u_{\mathrm{a}}^{2}=0.01$ and varying heat exchange coefficient $\alpha_{1}=\alpha_{\mathrm{r}} \kappa_{\mathrm{r}} / u_{\mathrm{a}}^{2}$. From left to right: stability boundary for $\alpha_{1}=0 \cdot 1, \alpha_{1}=0.05$, and $\alpha_{1}=0.01$. Stability region is located to the left of the curve.

Next, we discuss the effect of heat influx from the inert layer into the reactive layer (Figure 5). We again observe an $S$-shaped dependence that degenerates as $\alpha_{\mathrm{i}}$ increases because the larger heat influx allows for the high-temperature branch to persist for any $\alpha_{\mathrm{r}}$.

Figure 6 illustrates the effect of the ratio of the thermal diffusivities on the front temperature. The main conclusion here is that increasing the thermal diffusivity of the reactive layer relative to that of the inert layer facilitates high-temperature propagation and causes degeneration of the $S$-shaped behavior.

\section{Linear stability analysis}

We now turn to the stability analysis of the steady-state solution. We perturb the system about the basic state

$$
\begin{aligned}
& T_{\mathrm{r}}(x, t)=\widehat{T}_{\mathrm{r}}(x)+\widetilde{T}_{\mathrm{r}}(x) \exp (\omega t), \quad T_{\mathrm{i}}(x, t)=\widehat{T}_{\mathrm{i}}(x)+\widetilde{T}_{\mathrm{i}}(x) \exp (\omega t), \\
& \frac{\mathrm{d} \varphi}{\mathrm{d} t}=-\widehat{u}+\widetilde{\phi} \exp (\omega t) .
\end{aligned}
$$

Here $\omega$ is the growth rate of the perturbation. Substituting (31-33) in (7-8) we obtain differential equations for the perturbations as

$$
\begin{aligned}
& \kappa_{\mathrm{r}} \widetilde{T}_{\mathrm{r}}^{\prime \prime}-\widehat{u} \widetilde{T}_{\mathrm{r}}^{\prime}-\alpha_{\mathrm{r}}\left(\widetilde{T}_{\mathrm{r}}-\widetilde{T}_{\mathrm{i}}\right)-\omega \widetilde{T}_{\mathrm{r}}=-\widetilde{\phi} \widehat{T}_{\mathrm{r}}^{\prime}, \\
& \kappa_{i} \widetilde{T}_{\mathrm{i}}^{\prime \prime}-\widehat{u} \widetilde{T}_{\mathrm{i}}^{\prime}-\alpha_{\mathrm{i}}\left(\widetilde{T}_{\mathrm{i}}-\widetilde{T}_{\mathrm{r}}\right)-\omega \widetilde{T}_{\mathrm{i}}=-\widetilde{\phi} \widehat{T}_{\mathrm{i}}^{\prime},
\end{aligned}
$$

where a prime denotes the derivative with respect to $x$. These equations have to be solved ahead of the front $(x<0)$ and behind the front $(x>0)$ subject to the boundary conditions 


$$
\begin{array}{ll}
x=-\infty: & \widetilde{T}_{\mathrm{r}}=\widetilde{T}_{\mathrm{i}}=0, \\
x=\infty: & \widetilde{T}_{\mathrm{r}}=\widetilde{T}_{\mathrm{i}}=0
\end{array}
$$

and linearized matching conditions at the front $(x=0)$

$$
\begin{aligned}
& {\left[\widetilde{T}_{\mathrm{r}}\right]=\left[\widetilde{T}_{\mathrm{i}}\right]=0,} \\
& \kappa_{\mathrm{r}}\left[\frac{\mathrm{d} \widetilde{T}_{\mathrm{r}}}{\mathrm{d} x}\right]=q \widehat{u} \widetilde{M}_{\mathrm{b}}+q \widetilde{\phi}\left(M_{0}-\widehat{M}_{\mathrm{b}}\right), \quad\left[\frac{\mathrm{d} \widetilde{T}_{\mathrm{i}}}{\mathrm{d} x}\right]=0, \\
& -2 \widehat{u} \widetilde{\phi}=F^{\prime}\left(\widehat{T}_{\mathrm{b}}\right) \widetilde{T}_{\mathrm{b}}, \quad \widetilde{M}_{\mathrm{b}}=f^{\prime}\left(\widehat{T}_{\mathrm{b}}\right) \widetilde{T}_{\mathrm{b}} .
\end{aligned}
$$

We observe that a particular solution of the system (34), (35) is determined as $\left(\widetilde{\phi} \widehat{T}_{\mathrm{r}}^{\prime} / \omega\right.$, $\left.\widetilde{\phi} \widehat{T}_{\mathrm{i}}^{\prime} / \omega\right)$. Indeed, it can be verified by substituting the solution in (34), (35) and taking into account that

$$
\kappa_{\mathrm{r}} \widehat{T}_{\mathrm{r}}^{\prime \prime \prime}-\widehat{u} \widehat{T}_{\mathrm{r}}^{\prime \prime}-\alpha_{\mathrm{r}}\left(\widehat{T}_{\mathrm{r}}^{\prime}-\widehat{T}_{\mathrm{i}}^{\prime}\right)=0, \quad \kappa_{\mathrm{i}} \widehat{T}_{\mathrm{i}}^{\prime \prime \prime}-\widehat{u} \widehat{T}_{\mathrm{i}}^{\prime \prime}-\alpha_{\mathrm{i}}\left(\widehat{T}_{\mathrm{i}}^{\prime}-\widehat{T}_{\mathrm{r}}^{\prime}\right)=0,
$$

as follows from (13), (14). Thus, the solution $\widetilde{T}_{\mathrm{r}}, \widetilde{T}_{\mathrm{i}}$ can be represented in the form

$$
\widetilde{T}_{\mathrm{r}}=\frac{\widetilde{\phi} \widehat{T}_{\mathrm{r}}^{\prime}}{\omega}+\widetilde{T}_{\mathrm{rh}}, \quad \widetilde{T}_{\mathrm{i}}=\frac{\widetilde{\phi} \widehat{T}_{\mathrm{i}}^{\prime}}{\omega}+\widetilde{T}_{\mathrm{ih}},
$$

where $\widetilde{T}_{\text {rh }}, \widetilde{T}_{\text {ih }}$ is the homogeneous solution that is given by

$$
\begin{gathered}
\frac{\widetilde{T}_{\mathrm{rh}}(x)}{q\left(M_{0}-\widehat{M}_{\mathrm{b}}\right)}= \begin{cases}A_{2} \exp \left(\mu_{2} \widehat{u} x / \kappa_{\mathrm{r}}\right)+A_{3} \exp \left(\mu_{3} \widehat{u} x / \kappa_{\mathrm{r}}\right), & x<0 \\
A_{0} \exp \left(\mu_{0} \widehat{u} x / \kappa_{\mathrm{r}}\right)+A_{1} \exp \left(\mu_{1} \widehat{u} x / \kappa_{\mathrm{r}}\right), & x>0\end{cases} \\
\frac{\widetilde{T}_{\mathrm{ih}}(x)}{q\left(M_{0}-\widehat{M}_{\mathrm{b}}\right)}=\left\{\begin{array}{ll}
k_{2} A_{2} \exp \left(\mu_{2} \widehat{u} x / \kappa_{\mathrm{r}}\right)+k_{3} A_{3} \exp \left(\mu_{3} \widehat{u} x / \kappa_{\mathrm{r}}\right), & x<0 \\
k_{0} A_{0} \exp \left(\mu_{0} \widehat{u} x / \kappa_{\mathrm{r}}\right)+k_{1} A_{1} \exp \left(\mu_{1} \widehat{u} x / \kappa_{\mathrm{r}}\right), & x>0
\end{array} .\right.
\end{gathered}
$$

Here $A_{n}(n=0,1,2,3)$ are as yet undetermined amplitudes of the homogeneous solution, the quantities $k_{n}$ are given by

$$
k_{n}=1-\frac{\mu_{n}^{2}-\mu_{n}-\Omega}{\alpha_{\mathrm{r}}^{0}},
$$

where

$$
\Omega=\frac{\kappa_{\mathrm{r}}}{\widehat{u}^{2}} \omega
$$

is the nondimensional frequency of the perturbation, and $\mu_{n}$ are the roots of the characteristic equation

$$
\begin{gathered}
\kappa^{0} \mu^{4}-\left(1+\kappa^{0}\right) \mu^{3}+\left(1-\Omega\left(1+\kappa^{0}\right)-\alpha_{\mathrm{i}}^{0} / \kappa^{0}-\alpha_{\mathrm{r}}^{0} \kappa^{0}\right) \mu^{2} \\
+\left(2 \Omega+\alpha_{\mathrm{r}}^{0}+\alpha_{\mathrm{i}}^{0} / \kappa^{0}\right) \mu+\Omega^{2}+\Omega\left(\alpha_{\mathrm{r}}^{0}+\alpha_{\mathrm{i}}^{0} / \kappa^{0}\right)=0 .
\end{gathered}
$$

In what follows we will be interested in the stability boundary that separates the regions in the parameter space where the steady-state solution is stable from those where it is unstable. At the stability boundary $\Omega=$ is, $s \geq 0$. It can be shown that for such $\Omega$ the characteristic equation has two roots with positive real part (we denote them by $\mu_{2}$ and $\mu_{3}$ ), and two roots with negative real part $\left(\mu_{0}\right.$ and $\left.\mu_{1}\right)$. This explains why the solution in (42), (43), which must decay as $x \rightarrow \pm \infty$, contains two exponentials for $x>0$ and two exponentials for $x<0$. 
Next, we use the six matching conditions in $(38-40)$ and the condition that $\widetilde{T}_{\mathrm{b}}$ is the perturbation of the reactive temperature at $x=0$ to obtain a system of seven linear equations for the unknown amplitudes $A, \widetilde{\phi}$ and the temperature and the monomer concentrations $\widetilde{T}_{\mathrm{b}}$ and $\widetilde{M}_{\mathrm{b}}$ at the front. It is convenient first to translate the matching conditions (38-40) into matching conditions for $\widetilde{T}_{\mathrm{rh}}$ and $\widetilde{T}_{\mathrm{ih}}$. Substituting (41) in (38), (39) and using (40) we obtain

$$
\begin{aligned}
& {\left[\widetilde{T}_{\mathrm{rh}}\right]=\frac{\widetilde{\phi}}{\omega} \frac{q \widehat{u}}{\kappa_{\mathrm{r}}}\left(M_{0}-\widehat{M}_{\mathrm{b}}\right), \quad\left[\widetilde{T}_{\mathrm{hh}}\right]=0,} \\
& \kappa_{\mathrm{r}}\left[\widetilde{T}_{\mathrm{ih}}^{\prime}\right]=q \widetilde{\phi}\left(M_{0}-\widehat{M}_{\mathrm{b}}\right)\left(1+\frac{\widehat{u}^{2}}{\omega \kappa_{\mathrm{r}}}\right)+q \widehat{u} f^{\prime}\left(\widehat{T}_{\mathrm{b}}\right)\left(\widetilde{T}_{\mathrm{rh}}(+0)+\frac{\widetilde{\phi}}{\omega} \widehat{T}_{\mathrm{r}}^{\prime}(+0)\right), \\
& {\left[\widetilde{T}_{\mathrm{ih}}^{\prime}\right]=0, \quad-2 \widehat{u} \widetilde{\phi}=F^{\prime}\left(\widehat{T}_{\mathrm{b}}\right)\left(\widetilde{T}_{\mathrm{rh}}(+0)+\frac{\widetilde{\phi}}{\omega} \widehat{T}_{\mathrm{r}}^{\prime}(+0)\right) .}
\end{aligned}
$$

Substituting (42), (43) in the matching conditions we obtain the system of linear equations

$$
\begin{aligned}
& A_{0}+A_{1}-A_{2}-A_{3}=\frac{\Psi}{\Omega}, \\
& A_{0} \mu_{0}+A_{1} \mu_{1}-A_{2} \mu_{2}-A_{3} \mu_{3}=\frac{\Psi}{\Omega}(1+P Q)+\Psi+P\left(A_{0}+A_{1}\right), \\
& A_{0} k_{0}+A_{1} k_{1}-A_{2} k_{2}-A_{3} k_{3}=0, \quad A_{0} \mu_{0} k_{0}+A_{1} \mu_{1} k_{1}-A_{2} \mu_{2} k_{2}-A_{3} \mu_{3} k_{3}=0, \\
& -2 \Psi=Z\left(A_{0}+A_{1}+\frac{\Psi}{\Omega} Q\right)
\end{aligned}
$$

for $A_{0}, A_{1}, A_{2}, A_{3}$, and $\Psi=\widetilde{\phi} / \widehat{u}$. Here $P, Q$ and $Z$ are three groups of parameters that characterize the steady-state solution,

$$
\begin{aligned}
& P=q f^{\prime}\left(\widehat{T}_{\mathrm{b}}\right), \quad Q=\frac{\kappa_{\mathrm{r}} \widehat{T}_{\mathrm{r}}^{\prime}(+0)}{\widehat{u} q\left(M_{0}-\widehat{M}_{\mathrm{b}}\right)}, \\
& Z=q\left(M_{0}-\widehat{M}_{\mathrm{b}}\right) \frac{F^{\prime}\left(\widehat{T}_{\mathrm{b}}\right)}{F\left(\widehat{T}_{\mathrm{b}}\right)}=q\left(M_{0}-\widehat{M}_{\mathrm{b}}\right) \frac{\mathrm{d} \log \widehat{u}^{2}}{\mathrm{~d} \widehat{T}_{\mathrm{b}}} .
\end{aligned}
$$

Parameter $Z$ demonstrates how sensitive the propagation velocity is to changes in the front temperature. It is a standard stability parameter in most combustion and frontal polymerization stability analyses. Parameter $P$ is typical of most frontal polymerization problems. It arises due to the fact that polymerization conversion is incomplete, and shows how this incompleteness relates to the front temperature. Parameter $Q$ is specific of problems in which heat exchange with the environment occurs. Indeed, in the absence of such exchange the temperature in the steady-state would be zero behind the front so $Q$ would be zero. We remark that using the steady-state solution (20) results in $Q=v_{1} C_{1}$, i.e., it has the form

$$
Q=-\frac{\alpha_{\mathrm{r}}^{0}+\left(\nu_{3}-1\right)\left(\nu_{2}-1\right)}{\left(v_{1}-v_{3}\right)\left(v_{1}-v_{2}\right)} .
$$

The condition that the above system of linear equations has a nontrivial solution, i.e., the determinant of the system is equal to zero, yields the dispersion relation

$$
\left(\mu_{2}-\mu_{3}\right)\left(\mu_{0}-\mu_{1}\right)\left(\left(H_{1}+H_{2} Q\right) Z+H_{3} P+H_{4}\right)=0,
$$


where the coefficients $H_{1}, H_{2}, H_{3}, H_{4}$ are functions of $\mu_{0}, \mu_{1}, \mu_{2}, \mu_{3}, \Omega$ and $\alpha_{\mathrm{r}}^{0}$ given by

$$
\begin{aligned}
H_{1}= & \left(1+3 \Omega+\Omega^{2}+2 \alpha_{\mathrm{r}}^{0}+\alpha_{\mathrm{r}}^{0} \Omega\right)\left(\mu_{2}+\mu_{3}-\mu_{0}-\mu_{1}\right) \\
& +\left(\alpha_{\mathrm{r}}^{0}+2 \Omega+1\right)\left(\mu_{0}^{2}+\mu_{1}^{2}-\mu_{2}^{2}-\mu_{3}^{2}+\mu_{0} \mu_{1}-\mu_{2} \mu_{3}\right) \\
& +(\Omega+1)\left(\mu_{2}^{2} \mu_{0}-\mu_{2} \mu_{0}^{2}+\mu_{2}^{2} \mu_{1}-\mu_{2} \mu_{1}^{2}+\mu_{3}^{2} \mu_{1}-\mu_{3} \mu_{1}^{2}+\mu_{3}^{2} \mu_{0}-\mu_{3} \mu_{0}^{2}\right) \\
& +(\Omega+1)\left(\mu_{0} \mu_{2} \mu_{3}-\mu_{0} \mu_{1} \mu_{2}+\mu_{1} \mu_{2} \mu_{3}-\mu_{0} \mu_{1} \mu_{3}\right) \\
& +\mu_{2}^{2} \mu_{3}^{2}+\mu_{0} \mu_{1} \mu_{2} \mu_{3}-\mu_{2} \mu_{3}^{2} \mu_{0}+\mu_{2} \mu_{3} \mu_{0}^{2}-\mu_{2} \mu_{3}^{2} \mu_{1}+\mu_{2} \mu_{3} \mu_{1}^{2}-\mu_{2}^{2} \mu_{3} \mu_{0}-\mu_{2}^{2} \mu_{3} \mu_{1}, \\
H_{2}= & \left(\mu_{3}-\mu_{1}\right)\left(\mu_{3}-\mu_{0}\right)\left(\mu_{2}-\mu_{1}\right)\left(\mu_{2}-\mu_{0}\right), \quad H_{4}=2 \Omega H_{2}, \\
H_{3}= & -2 \Omega\left\{\left(1+\Omega+\alpha_{\mathrm{r}}^{0}\right)\left(\mu_{2}+\mu_{3}-\mu_{0}-\mu_{1}\right)+\mu_{0}^{2}+\mu_{1}^{2}-\mu_{2}^{2}-\mu_{3}^{2}+\mu_{0} \mu_{1}-\mu_{2} \mu_{3}\right. \\
& +\mu_{2}^{2} \mu_{0}-\mu_{2} \mu_{0}^{2}+\mu_{2}^{2} \mu_{1}-\mu_{2} \mu_{1}^{2}+\mu_{3}^{2} \mu_{1}-\mu_{3} \mu_{1}^{2}+\mu_{3}^{2} \mu_{0}-\mu_{3} \mu_{0}^{2} \\
& \left.+\mu_{0} \mu_{2} \mu_{3}-\mu_{0} \mu_{1} \mu_{2}+\mu_{1} \mu_{2} \mu_{3}-\mu_{0} \mu_{1} \mu_{3}\right\} .
\end{aligned}
$$

Note that $\Omega=0$ is always a solution of the dispersion relation due to translational invariance of the problem. Indeed, substituting $\Omega=0$ in (46), taking into account that, for $\Omega=0$, the characteristic roots $\mu$ become the characteristic roots $v$ (which can be seen by comparing the two characteristic equations), i.e., $\mu_{0}=0, \mu_{1}=v_{1}, \mu_{2}=v_{2}, \mu_{3}=v_{3}$, and, finally, using (45) reduces the left-hand side of the dispersion relation to

$$
\begin{gathered}
-v_{1} Z\left(v_{2}-v_{3}\right)\left(v_{2}+v_{3}-v_{1}\right)\left\{\left(\alpha_{\mathrm{r}}^{0}+1\right)\left(v_{1}+v_{2}+v_{3}\right)\right. \\
\left.-\left(v_{1} v_{2}+v_{1} v_{3}+v_{2} v_{3}\right)+v_{1} v_{2} v_{3}-2 \alpha_{\mathrm{r}}^{0}-1\right\} .
\end{gathered}
$$

This expression is equal to zero because the last factor is equal to zero, which can be checked using the Vieta formulas for a cubic equation.

Monotone stability boundaries $\Omega=0$ correspond to the turning points in the Figures 4-6 and result in the instability of the intermediate solution branches.

In order to determine the oscillatory stability boundary, we set $\Omega=$ is, $(s>0)$, in the dispersion relation (46). Separating the real and the imaginary parts in the resulting equation, we obtain two real equations, from which $Z$ and $P$ can be found as functions of $s$ as

$$
Z=\frac{\mathfrak{I m}\left(H_{3} \bar{H}_{4}\right)}{\mathfrak{I m}\left(\left(H_{1}+H_{2} Q\right) \bar{H}_{4}\right)}, \quad P=\frac{\mathfrak{I m}\left(H_{4}\left(\bar{H}_{1}+\bar{H}_{2} Q\right)\right)}{\mathfrak{I m}\left(\left(H_{1}+H_{2} Q\right) \bar{H}_{4}\right)},
$$

where the bar denotes the complex conjugate. Thus, the above equations parametrically (with $s$ being the parameter) determine the stability boundaries in the $(Z, P)$-plane. Analysis of these equations shows two striking results. First, the stability boundaries are mainly determined by the parameters $Z$ and $P$. The boundaries are not sensitive to thermophysical parameter values for the parameter ranges under study, with the sole exception of $\alpha_{\mathrm{r}}$, the heat-exchange parameter in the reactive layer. Second, the boundaries are given by almost perfect straight lines. Figure 7 illustrates these results. Stability regions are located to the left of the corresponding stability boundaries, and we observe that increasing $\alpha_{\mathrm{r}}$ reduces the stability region. This result is consistent with the stability analysis of nonadiabatic polymerization fronts in [12], which showed that heat losses destabilize front propagation. We remark that changing other thermophysical parameters, namely, $\kappa^{0}$ and $\alpha_{i}$, has not resulted in any noticeable changes in the stability boundaries. 


\section{Conclusion}

We have considered the steady propagation of a polymerization front in a sandwich-type twolayer model. One layer initially contains reactants that can undergo polymerization. The other layer consists of an inert material. Heat exchange is possible between the two layers and thus the presence of the inert layer can significantly affect propagation of the polymerization wave.

The solution of the problem is not necessarily unique. We observe a turning point in the graphical representation of the front temperature (see Figures 4-6) and beyond this point the steady-state solution is no longer unique. There are three different uniformly propagating waves that exist for the same parameter values. This is a source of hysteresis as the system can jump between the upper and lower branches as the parameters are varied. Nonuniqueness of solution is also observed in the case of a pure polymerization problem, i.e., without an additional layer [11]. However, in this case only up to two solutions can exist, one of which is necessarily unstable, so that hysteresis cannot occur. We find that for sufficiently large heatexchange parameter in the reactive layer, the frontal polymerization reaction is quenched, or at best, significantly inhibited. On the other hand, increasing the heat-exchange parameter in the inert layer facilitates propagation of high-temperature fronts.

We proceeded to perform a linear stability analysis of the steady-state solutions. In the case of multiple solutions, the intermediate branch is always unstable. The upper and lower branches can be either stable or unstable depending on the parameter values. The same is true in the case of a single steady-state solution. Our analysis indicated that the front stability is rather insensitive to the thermo-physical parameters of the problem, except for the heat-exchange parameter in the reactive layer. Increasing this parameter has a significant destabilizing effect. In general, the overall linear stability of the system is promoted by incomplete monomer conversion by the frontal polymerization process occurring within the reacting layer. The same effect of incomplete monomer conversion has been observed in the case of a pure polymerization problem [12].

\section{Acknowledgement}

This research has been supported by NSF grant CTS-0138712.

\section{References}

1. N.M. Chechilo, R.J. Khvilivitskii and N.S. Enikolopyan, On the phenomenon of polymerization reaction spreading. Dokl. Akad. Nauk SSSR 204 (1972) 1180-1181.

2. M.F. Perry and V.A. Volpert, Self-propagating free-radical binary frontal polymerization. J. Engng. Math. 49 (2004) 359-372.

3. K.G. Shkadinskii and P.M. Krishenik, Steady combustion front in a mixture of fuel and inert material. Combust. Explos. Shock Waves 21 (1985) 176-180.

4. G.G. Odian, Principles of Polymerization, 3rd edition. New York: Wiley-Interscience (1991) 768 pp.

5. C.A. Spade and V.A. Volpert, On the steady state approximation in thermal free radical frontal polymerization. Chem. Engng. Sci. 55 (2000) 641-654.

6. G.B. Manelis, L.P. Smirnov and N.I. Peregudov, Nonisothermal kinetics of polymerization processes. Finite cylindrical reactor. Combust. Explos. Shock Wave 13 (1977) 389-393.

7. A.G. Merzhanov and B.I. Khaikin, Theory of combustion waves in homogeneous media. Prog. Ener. Combust. Sci. 14 (1988) 1-98.

8. S.B. Margolis and B.J. Matkowsky, Flame propagation with a sequential reaction mechanism. SIAM J. Appl. Math. 42 (1982) 1175-1188.

9. V.A. Volpert and P.M. Krishenik, Nonsteady propagation of combustion waves in a system of successive reactions with endothermal stages. Combust. Explos. Shock Waves 22 (1986) 285-292. 


\section{D.M.G. Comissiong et al.}

10. D.M.G. Comissiong, L.K. Gross and V.A. Volpert, Nonlinear dynamics of frontal polymerization with autoacceleration. J. Engng. Math. 53 (2005) 59-78.

11. P.M. Goldfeder and V.A. Volpert, Nonadiabatic frontal polymerization. J. Engng. Math. 34 (1998) 301-318.

12. C.A. Spade and V.A. Volpert, Linear stability analysis of non-adiabatic free-radical polymerization waves. Combust. Theory Model. 5 (2001) 21-39. 